\title{
Oxygen on Ni(111): A multiple-scattering analysis of the near-edge x-ray-absorption fine structure
}

\author{
M. Pedio, ${ }^{*}$ L. Becker, B. Hillert, S. D’Addato, ${ }^{\dagger}$ and J. Haase \\ Fritz-Haber-Institut der Max-Planck-Gesellschaft, Faradayweg 4-6, D-1000 Berlin 33, West Germany
}

(Received 31 October 1989; revised manuscript received 2 January 1990)

\begin{abstract}
Oxygen chemisorption and oxide formation on a Ni(111) surface have been monitored by using the near-edge $x$-ray-absorption fine-structure technique. The adsorption site of oxygen in the $p(2 \times 2)$ and $(\sqrt{3} \times \sqrt{3}) R 30^{\circ}$ superstructures has been determined by a multiple-scattering analysis. In both structures the oxygen occupies a threefold-coordinated fcc site with a nearest-neighbor $\mathrm{O}-\mathrm{Ni}$ bond length of $1.85 \pm 0.05 \AA$ on a $\mathrm{Ni}(111)$ surface relaxed outwards by $\sim 0.15 \AA$.
\end{abstract}

The near-edge $x$-ray-absorption fine-structure (NEXAFS) technique is known to provide valuable information on the geometric structure of atomic and molecular adsorbates on surfaces. For adsorbed molecular species NEXAFS and surface extended x-ray-absorption fine-structure (SEXAFS) results are complementary. For atomic adsorbates, on the other hand, a multiplescattering (MS) analysis of NEXAFS (Refs. 1-4) yields the same structural parameters as SEXAFS: adsorbatesubstrate interlayer spacings (i.e., bond lengths), adsorption sites, and adsorbate-induced surface reconstructions and relaxations. Because of its higher experimental accuracy and reliability the SEXAFS technique is generally favored for atomic-adsorbate studies. SEXAFS spectra, however, are dominated by a small number (often only one or two) of nearest-neighbor (NN) "shells" of atoms which is due to the $1 / R^{2}$ dependence of the signal, to Debye-Waller-like effects, and also to the finite mean free path of the emitted photoelectron. Because of the longer mean free path closer to threshold and consequent MS of the photoelectron the "cluster" of scatterers contributing to the NEXAFS is generally larger. It may thus happen that NEXAFS yields more structural information than SEXAFS. Moreover, SEXAFS is difficult to apply to systems with interfering absorption edges and may suffer from sensitivity problems for submonolayer coverages. In this paper we show that a MS analysis of the NEXAFS of the $p(2 \times 2)$ and $(\sqrt{3} \times \sqrt{3}) R 30^{\circ}$ structures of oxygen on $\mathrm{Ni}(111)$ indicates a threefold-coordinated fcc adsorption site for both structures and excludes a hcp site. This information was not available up to now and cannot unambiguously be obtained with SEXAFS.

The O/Ni(111) system has been widely studied. ${ }^{5-21}$ Oxygen exposure gives rise consecutively to $p(2 \times 2)$ and $(\sqrt{3} \times \sqrt{3}) R 30^{\circ}$ low-energy electron diffraction (LEED) patterns which correspond to coverages of 0.25 monolayer (ML) and 0.33 ML, respectively. From a number of different techniques it is known that at higher oxygen exposures oxide islands form which finally grow laterally to coalescence at about two to three layers of $\mathrm{NiO}^{5}$. Early LEED studies ${ }^{7}$ on the $p(2 \times 2)-\mathrm{O} / \mathrm{Ni}(111)$ system suggested a threefold-coordinated adsorption site as it is shown in Fig. 1 with a nearest-neighbor $\mathrm{O}-\mathrm{Ni}$ distance of $R=1.88 \pm 0.06 \AA$, which corresponds to a perpendicular distance of the oxygen atoms from the first $\mathrm{Ni}$ layer of $z=1.21 \pm 0.09 \AA$. A distinction between fcc and hcp sites, however, was not possible. In a subsequent study ${ }^{12}$ it was demonstrated that only one such site is taken up at coverages below $0.27 \mathrm{ML}$, while above $0.27 \mathrm{ML}$ both types of site could be occupied simultaneously. Ion scattering experiments ${ }^{15}$ indicated an outward relaxation of the three nearest $\mathrm{Ni}$ neighbors and an outward relaxation of the whole first Ni layer by $\Delta d_{12}=0.15 \AA$ (cf. Fig. 1) for the $p(2 \times 2)$ and $(\sqrt{3} \times \sqrt{3}) R 30^{\circ}$ structures, respectively, but could not distinguish either between fcc and hcp sites. However, only one type of site should be occu-
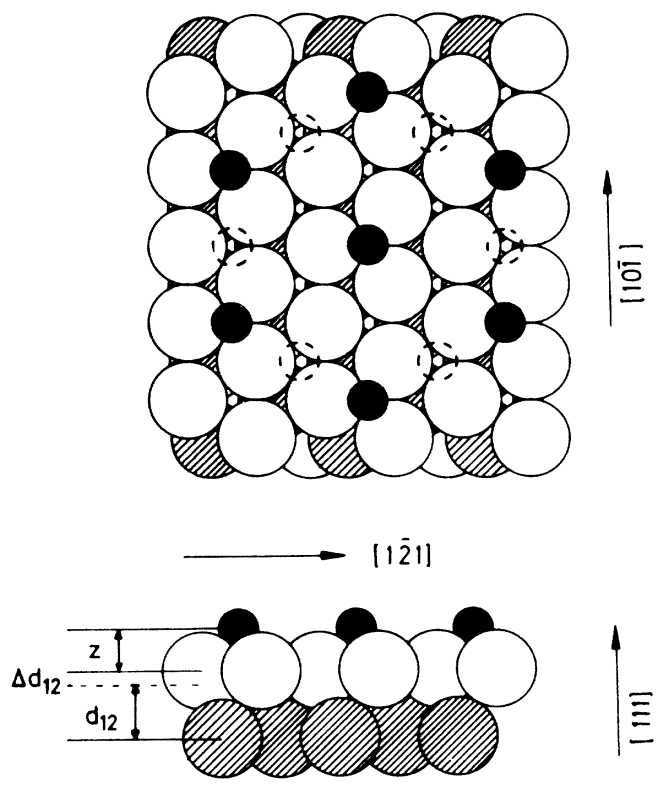

FIG. 1. Schematic geometry of the $p(2 \times 2)-\mathrm{O} / \mathrm{Ni}(111)$ system in a top view (above) and a side view (below) with the oxygen atoms (solid circles) in the threefold-coordinated fcc sites. $d_{12}$ represents the bulk $\mathrm{Ni}$ interlayer spacing, $\Delta d_{12}$ an outward relaxation of the first $\mathrm{Ni}$ layer. The dashed circles in the top view mark the positions of the oxygen atoms in the $(\sqrt{3} \times \sqrt{3}) R 30^{\circ}-\mathrm{O} / \mathrm{Ni}(111)$ system (threefold-coordinated fcc sites). 
pied for both structures. A recent SEXAFS study ${ }^{21}$ on both systems determined the NN O-Ni distance as $R=1.86 \pm 0.03 \AA$, confirmed the threefold coordination of the adsorbed oxygen, and favored a fec site on an outward relaxed surface. For both structures we find best agreement between experiment and MS NEXAFS calculations for a threefold-coordinated fcc site above $\mathrm{Ni}$ surface atoms relaxed outwards by $0.15 \AA$ with a NN O-Ni distance of $1.85 \pm 0.05 \AA$ in remarkable agreement with LEED, ion scattering, and SEXAFS results.

The experiments were performed at the electron storage ring BESSY using the SX-700 monochromator. ${ }^{22}$ NEXAFS data were taken above the oxygen $K$ edge in the partial electron yield mode for $x$-ray incidence angles of $\theta=90^{\circ}$ (normal incidence; $\mathbf{E}$ vector parallel to the surface) and $\theta=20^{\circ}$ (near-grazing incidence) with respect to the surface. The Ni(111) sample was cleaned by successive cycles of argon-ion bombardment, annealing at 750 $\mathrm{K}$, and heating to $1000 \mathrm{~K}$. Oxygen exposures and measurements were performed at room temperature with one exception: the $(\sqrt{3} \times \sqrt{3}) R 30^{\circ}$ LEED pattern exhibited maximum contrast around $3 \mathrm{~L}$ [1 langmuir $(\mathrm{L})=10^{-6}$ Torr sec] exposure at $200 \mathrm{~K}$. The bulk NiO sample was
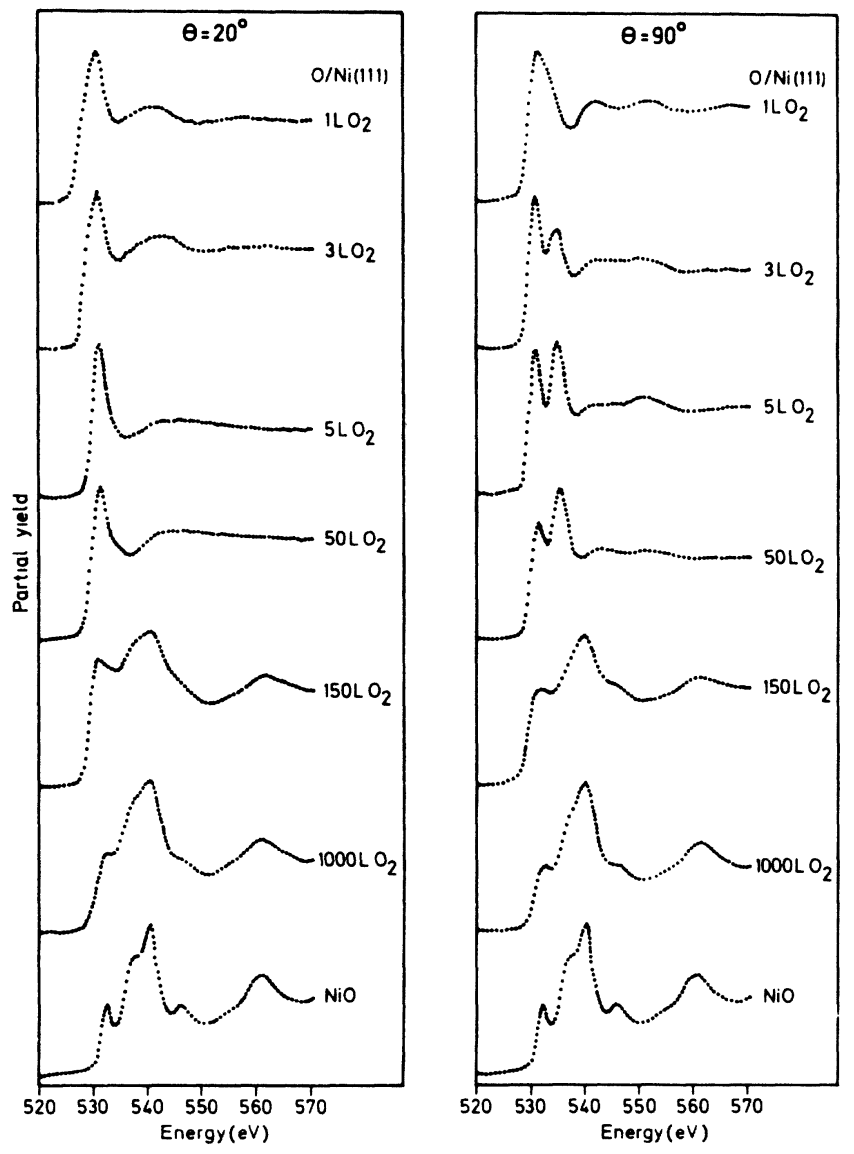

FIG. 2. Oxygen $K$-edge NEXAFS spectra taken in the partial electron yield mode at near-grazing $\left(\theta=20^{\circ}\right)$ and at normal $\left(\theta=90^{\circ}\right) \mathrm{x}$-ray incidence for $\mathrm{O}$ on $\mathrm{Ni}(111)$ with the oxygen exposure as parameter and for NiO. Exposures of 1 and $3 \mathrm{~L}$ correspond to the $p(2 \times 2)$ and $(\sqrt{3} \times \sqrt{3}) R 30^{\circ}$ structure, respectively. [1 langmuir $(\mathrm{L}) \equiv 10^{-6}$ Torr sec.] prepared from $\mathrm{NiO}$ powder.

Oxygen $K$-edge NEXAFS spectra taken at neargrazing and normal incidence for the progressive oxidation of $\mathrm{Ni}(111)$ are shown in Fig. 2. At low oxygen exposures there is a strong polarization dependence. Up to an exposure of $50 \mathrm{~L}$ practically only the normal-incidence data change, indicating structural changes mainly parallel to the surface. Between 50 and $150 \mathrm{~L}$ threedimensional oxide formation occurs which now also results in a change of the near-grazing-incidence data and in a decreased polarization dependence. At an exposure of $1000 \mathrm{~L}$, finally, all structures seen in the NEXAFS of $\mathrm{NiO}$ are present with approximately the same relative intensities indicating a uniform $\mathrm{NiO}$ film on the surface. As can be seen from Fig. 2 NEXAFS is an instructive means for monitoring structural changes during the oxidation of $\mathrm{Ni}(111)$. Detailed structural information for the $p(2 \times 2)$ and $(\sqrt{3} \times \sqrt{3}) R 30^{\circ}$ structures was obtained by calculating NEXAFS spectra and comparing them to the experimental curves.

The calculations were performed with the program ICXANES (Ref. 23) which is based upon a cluster method and includes full MS. They have been checked for convergence in angular momentum and cluster size. The phase shifts are calculated from one-electron muffin-tin potentials constructed according to the Mattheis prescription ${ }^{24}$ for bulk $\mathrm{Ni}$ and for the oxygen atoms in a specific surface arrangement. The muffin-tin radii were chosen according to the Norman criterium. ${ }^{25}$ Damping was included via a $1-\mathrm{eV}$ imaginary part added to the energy of the outgoing electron. Several other potentials were tested, e.g., with the atoms in the $\mathrm{NiO}$ configuration, leading to substantially similar spectra to those presented here. Furthermore, good agreement was obtained between calculations of the NEXAFS of the $(2 \times 1)-\mathrm{O} / \mathrm{Ni}(110)$ system using our phase shifts and those performed by Lindner and Somers. ${ }^{4}$ For both the $p(2 \times 2)$ and $(\sqrt{3} \times \sqrt{3}) R 30^{\circ}$ structures the cluster included up to six shells of scatterers which corresponds to around 40 atoms up to a radial distance of $\sim 5.5 \AA$ from the central $\mathrm{O}$ atom. Each shell contained a set of atoms having a constant (within $0.5 \AA$ ) radial distance from the central atom. Calculations were performed for twofold bridge and threefold-coordinated fcc and hcp sites. For each geometry the NN O-Ni distance, the amount of surface relaxation as well as the $\mathrm{Ni}-\mathrm{Ni}$ distance on the surface was varied.

In Figs. 3 and 4 we compare the experimental $\mathrm{O} K$ edge NEXAFS spectra for the $p(2 \times 2)$ - and $(\sqrt{3} \times \sqrt{3}) R 30^{\circ}-\mathrm{O} / \mathrm{Ni}(111)$ systems, respectively, with the best fits of full MS calculations for both the threefold-coordinated fcc and hcp sites involving a NN $\mathrm{O}-\mathrm{Ni}$ distance of $1.85 \AA$, an outward relaxation of the surface $\mathrm{Ni}$ layer of $0.15 \AA$, and the bulk value for the $\mathrm{Ni}$ $\mathrm{Ni}$ distance on the surface. Calculated and measured NEXAFS spectra have been aligned to check the accuracy of the calculations in accounting for relative peak positions. The calculations for the twofold bridge site (not shown here) indicate that this site can be excluded as a possible adsorption site. For both structures the fcc site reproduces the experimental normal-incidence spectrum 

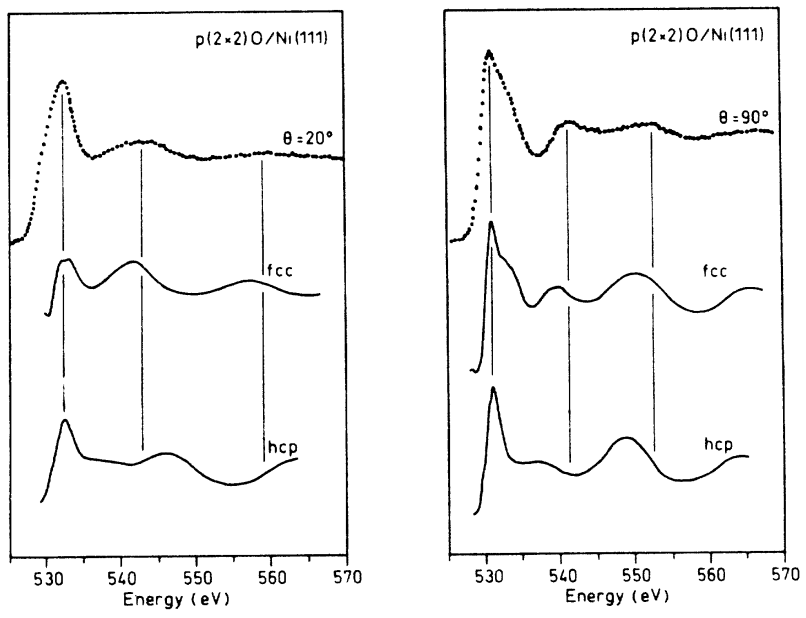

FIG. 3. Oxygen $K$-edge NEXAFS spectra for $p(2 \times 2)$ $\mathrm{O} / \mathrm{Ni}(111)$ taken in the partial electron yield mode at neargrazing $\left(\theta=20^{\circ}\right)$ and at normal $\left(\theta=90^{\circ}\right) \mathrm{x}$-ray incidence (dotted lines) and best fits of NEXAFS calculations (solid lines) for threefold-coordinated fcc and hep sites with a NN O-Ni distance of $1.85 \AA$ and an outward relaxation of the first Ni layer by $0.15 \AA$.

quite well with the fit for $(\sqrt{3} \times \sqrt{3}) R 30^{\circ}$ being better than for $p(2 \times 2)$. The maximum difference between calculated and experimental peak positions is less than $3 \mathrm{eV}$ for the fcc site and larger for the hcp site. Moreover, the structure some $\mathrm{eV}$ above the edge which appears as a shoulder in the $p(2 \times 2)$ and as a separate peak in the $(\sqrt{3} \times \sqrt{3}) R 30^{\circ}$ normal-incidence spectrum is much better reproduced by the fcc than by the hcp site. The comparison between calculated and experimental spectra for near-grazing incidence shows conclusively that only the threefold-coordinated fcc site is compatible with the data for both the $p(2 \times 2)$ and $(\sqrt{3} \times \sqrt{3}) R 30^{\circ}$ structures, i.e., the hcp site must be excluded. From numerous calculations the experimental accuracies for the $\mathrm{NN} \mathrm{O-Ni}$
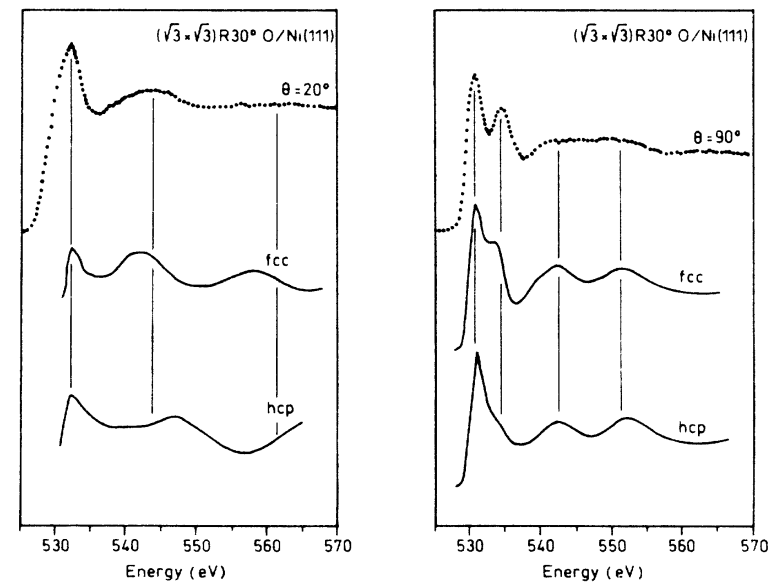

FIG. 4. Oxygen $K$-edge NEXAFS spectra for $(\sqrt{3} \times \sqrt{3}) R 30^{\circ}-\mathrm{O} / \mathrm{Ni}(111)$ taken in the partial electron yield mode at near-grazing $\left(\theta=20^{\circ}\right)$ and at normal $\left(\theta=90^{\circ}\right) \mathrm{x}$-ray incidence (dotted lines) and best fits of NEXAFS calculations (solid lines) for threefold-coordinated fcc and hep sites with a NN O-Ni distance of $1.85 \AA$ and an outward relaxation of the first Ni layer by $0.15 \AA$. distance and the surface relaxation could be assessed as follows: $R_{\mathrm{O}-\mathrm{Ni}}=1.85 \pm 0.05 \AA$ and $\Delta d_{12}=+0.15_{-0.15}^{+0.10} \AA$. Within experimental errors the NEXAFS results agree with LEED, ion scattering, and SEXAFS data and provide new information about the type of threefold coordination ( $\mathrm{fcc}$ ). The agreement between experiment and calculations for fcc sites is not perfect as can be seen from Figs. 3 and 4. Peak positions deviate by up to $3 \mathrm{eV}$ and the relative amplitudes for near-grazing incidence do not match very well. This is in part due to the fact that the program does not include a surface barrier (cf. Ref. 3) and the resulting loss of photoelectron current for energies below the work function. It is interesting to note that the work-function increase due to low oxygen exposure is much bigger on $\mathrm{Ni}(111)$ compared with $\mathrm{Ni}(100)$ and $\mathrm{Ni}(110)$ (Ref. 6) and also that it measures $0.8 \mathrm{eV}$ for the $p(2 \times 2)-\mathrm{O} / \mathrm{Ni}(111)$ system compared with $0.35 \mathrm{eV}$ for the $(\sqrt{3} \times \sqrt{3}) R 30^{\circ}-\mathrm{O} / \mathrm{Ni}(111)$ system. ${ }^{17}$ This might explain why the overall agreement between experiment and calculations for the systems $\mathrm{O} / \mathrm{Ni}(1.00)$ (Ref. 1) and $\mathrm{O} / \mathrm{Ni}(110)$ (Ref. 3) is better than for $\mathrm{O} / \mathrm{Ni}(111)$ and why the best fit for the $(\sqrt{3} \times \sqrt{3}) R 30^{\circ}$ structure is better than that for the $p(2 \times 2)$ structure.

Figure 5 shows a characteristic set of full MS calculations for the $(\sqrt{3} \times \sqrt{3}) R 30^{\circ}$ structure and the fcc site in which the number of shells in the cluster is successively increased together with a calculation based on the singlescattering (SS) approximation. For normal incidence the calculation with four shells is almost identical to the fiveand six-shell cluster result which reproduces the experimental curve very well. Inclusion of the fourth shell which contains neighboring $\mathrm{O}$ atoms results in an increase of the intensity roughly $5 \mathrm{eV}$ above the edge. This also explains the growth of this peak due to increased oxygen exposure during the initial stages of oxidation (cf.
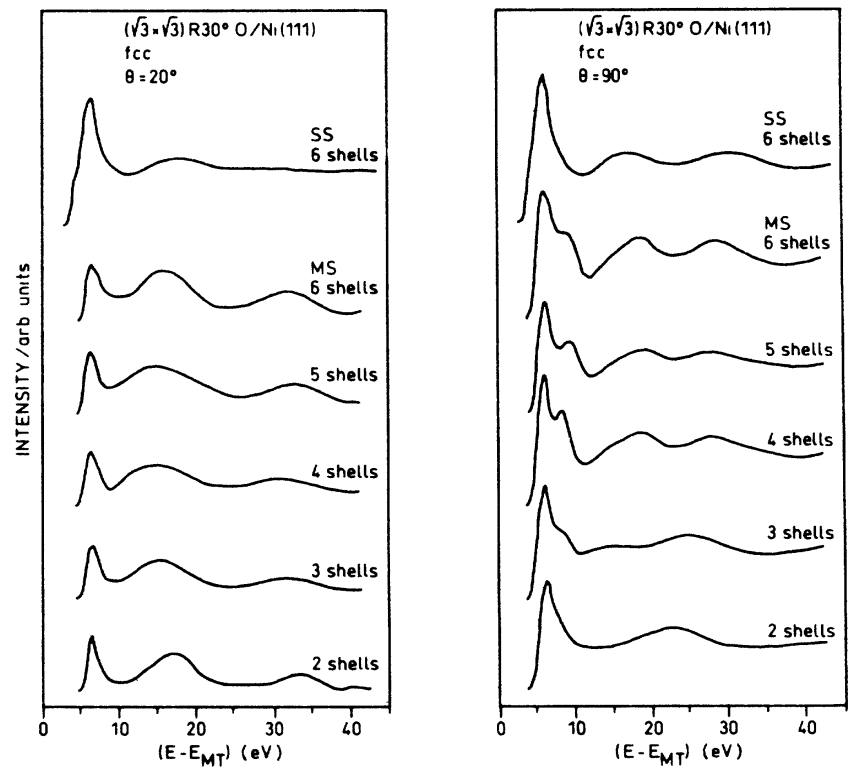

FIG. 5. Multiple-scattering (MS) NEXAFS calculations for $(\sqrt{3} \times \sqrt{3}) R 30^{\circ}-\mathrm{O} / \mathrm{Ni}(111)$ for near-grazing $\left(\theta=20^{\circ}\right)$ and normal $:\left(\theta=90^{\circ}\right) \mathrm{x}$-ray incidence displaying the effect of including more shells. The topmost curves use six shells in a single-scattering (SS) approximation. 
Figs. 3 and 4 and Fig. 2). Part of the intensity $\sim 5 \mathrm{eV}$ above the edge, however, is already present in the threeshell result of Fig. 5. Calculations omitting particular distances (instead of whole shells) showed that it is due to the third-nearest neighbor at a distance of $\sim 3.5 \AA$ which is only present for a fcc site. The same distance is also mainly responsible for the second peak roughly $30 \mathrm{eV}$ above the edge in the near-grazing-incidence data. As it is the position of this peak for $\theta=20^{\circ}$ and the structure $\sim 5 \mathrm{eV}$ above the edge for $\theta=90^{\circ}$ which constitute the differences in the calculations for a fcc and a hcp site (cf. Figs. 3 and 4) we have been able to easily distinguish between these two types of threefold coordination. Figure 5 also demonstrates that the overall profile of the SS calculation beyond $10 \mathrm{eV}$ above the edge is in qualitative agreement with the MS results whereas the structure $\sim 5$ $\mathrm{eV}$ above the edge seen in the MS calculation and in the experiment is lost. This is in agreement with previous applications of the ICXANES program to NEXAFS calculations for other atomic adsorbate systems. ${ }^{2-4}$

Finally, we made use of the possibility (provided by the program) of discriminating between intershell MS paths with pairs of atoms lying approximately collinear with the exicted atom and all other MS paths (intrashell). In Fig. 6 calculations with only intershell MS events are compared with full MS results for the $p(2 \times 2)-\mathrm{O} / \mathrm{Ni}(111)$ structure. Whereas the intershell MS calculation for $\theta=20^{\circ}$ reproduces the experimental spectrum quite well, there are distinct differences for $\theta=90^{\circ}$. The intensity $\sim 5 \mathrm{eV}$ above the edge showing up as a shoulder in the experiment and in the full MS calculation is strongly depressed when only intershell events are taken into account. This could indicate an anisotropy in the MS processes for this system and shows, on the other hand, that neglect of intrashell MS results in experimentally distinguishable differences in the calculated NEXAFS, as was already found for $\mathrm{NiO}^{26}$ A calculation with full MS up to $R \sim 3.5 \AA$ and only intershell MS beyond is practically identical with the full MS result indicating that intershell MS events dominate at higher distances.

A comparison between the NEXAFS spectra for $\mathrm{O} / \mathrm{Ni}(111), \mathrm{O} / \mathrm{Ni}(100),{ }^{1}$ and $\mathrm{O} / \mathrm{Ni}(110)$ (Ref. 4) reveals striking similarities for near-grazing incidence and differences for normal incidence. This can be explained in a SS picture. The near-grazing-incidence data are dominated by $\mathrm{O}-\mathrm{Ni}$ distances which are rather similar for all three systems $(1.85-1.98 \AA)$. Therefore, not only the overall structure, but also the separations of the dom-
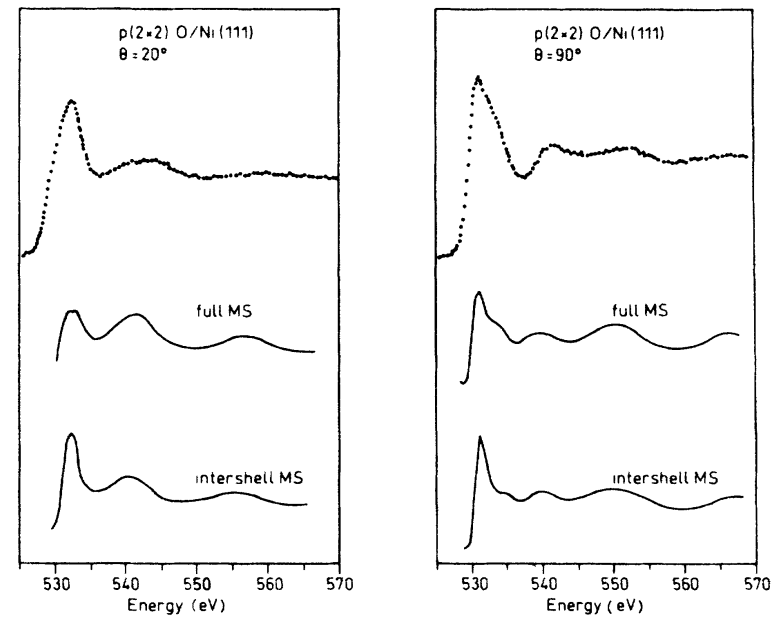

FIG. 6. Oxygen $K$-edge NEXAFS spectra for $p(2 \times 2)$ $\mathrm{O} / \mathrm{Ni}(111)$ taken at near-grazing $\left(\theta=20^{\circ}\right)$ and normal $\left(\theta=90^{\circ}\right)$ $\mathrm{x}$-ray incidence (dotted) lines and NEXAFS calculations (solid lines) using full multiple scattering and only intershell MS events.

inant peaks are rather similar. For normal incidence there is strong interference with the effective O-O distances which can be rather different for the systems in question [and depend on the azimuthal orientation of the E vector for $\mathrm{O} / \mathrm{Ni}(110)]$. The normal-incidence data have in common an extra peak between 5 and $10 \mathrm{eV}$ above the edge which is mainly due to the O-O interaction. The intensity of this peak depends on the O-O distance and on the effective coordination number.

In summary, our results show that NEXAFS studies are a sensitive monitor for structural changes at surfaces. They demonstrate that for simple systems MS calculations can provide detailed structural features of local adsorbate geometry and thus yield information which for exceptional cases may outweigh that obtained with SEXAFS.

This work has been funded by the German Federal Minister of Research and Technology (BMFT) under Contract No. 05390-FX-B2. We thank Th. Lindner and D. D. Vvedensky for fruitful discussions and G. Jäkisch for technical assistance throughout the measurements.
*Also at Istituto di Struttura della Materia del Consiglio Nazionale delle Ricerche, I-00044 Frascati, (Roma), Italy.

${ }^{\dagger}$ Also at Università degli Studi di Roma, La Sapienza, I-00185 Roma, Italy.

${ }^{1}$ D. Norman, J. Stöhr, R. Jaeger, P. J. Durham, and J. B. Pendry, Phys. Rev. Lett. 51, 2052 (1983).

${ }^{2}$ U. Döbler, K. Baberschke, D. D. Vvedensky, and J. B. Pendry, Surf. Sci. 178, 679 (1986).

${ }^{3}$ D. D. Vvedensky, J. B. Pendry, U. Döbler, and K. Baberschke, Phys. Rev. B 35, 7756 (1987).
${ }^{4}$ Th. Lindner and J. Somers, Phys. Rev. B 37, 10039 (1988).

${ }^{5}$ P. H. Holloway and J. B. Hudson, Surf. Sci. 43, 141 (1974).

${ }^{6}$ J. E. Demuth and T. N. Rhodin, Surf. Sci. 45, 249 (1974).

${ }^{7}$ P. M. Marcus, J. E. Demuth, and D. W. Jepsen, Surf. Sci. 53, 501 (1975).

${ }^{8}$ H. Conrad, G. Ertl, J. Küppers, and E. E. Latta, Solid State Commun. 17, 497 (1977).

${ }^{9}$ P. R. Norton, R. L. Tapping, and J. W. Goodale, Surf. Sci. 65, 13 (1977).

${ }^{10}$ T. W. Capehart and T. N. Rhodin, J. Vac. Sci. Technol. 16, 
594 (1979).

${ }^{11}$ L. D. Roelofs, T. L. Einstein, P. E. Hunter, A. R. Kortan, R. L. Park, and R. M. Roberts, J. Vac. Sci. Technol. 17, 231 (1980).

${ }^{12}$ L. D. Roelofs, A. R. Kortan, T. L. Einstein, and R. L. Park, Phys. Rev. Lett. 46, 1465 (1981).

${ }^{13}$ P. H. Holloway, J. Vac. Sci. Technol. 18, 653 (1981).

${ }^{14}$ A. R. Kortan and R. L. Park, Phys. Rev. B 23, 6340 (1981).

${ }^{15}$ T. Narusawa, W. M. Gibson, and E. Törnqvist, Phys. Rev. Lett. 47, 417 (1981); Surf. Sci. 114, 331 (1982).

${ }^{16}$ H.-T. Liu, A. F. Armitage, and D. P. Woodruff, Surf. Sci. 114, 431 (1982).

${ }^{17}$ D. F. Mitchell and M. J. Graham, Surf. Sci. 114, 546 (1982).

${ }^{18}$ F.-J. Himpsel and Th. Fauster, Phys. Rev. Lett. 49, 1583
(1982).

${ }^{19}$ W. Altmann, K. Desinger, M. Donath. V. Dose, A. Goldmann, and H. Scheidt, Surf. Sci. 151, L185 (1985).

${ }^{20}$ L. S. Caputi, S. L. Jiang, R. Tucci, and A. Amodeo, Surf. Sci. 211/212, 120 (1989).

${ }^{21}$ B. Hillert, L. Becker, M. Pedio, and J. Haase (unpublished).

${ }^{22}$ H. Petersen, Opt. Commun. 40, 402 (1982).

${ }^{23}$ D. D. Vvedensky, D. K. Saldin, and J. B. Pendry, Comput. Phys. Commun. 40, 421 (1986).

${ }^{24}$ L. F. Mattheiss, Phys. Rev. 134, A970 (1964).

25J. C. Norman, Mol. Phys. 31, 1191 (1976).

${ }^{26}$ D. D. Vvedensky and J. B. Pendry, Phys. Rev. Lett. 54, 2725 (1985). 